\title{
Novel Integrated Approaches for the Detection and Management of Type-2 Diabetes
}

\author{
Gundu H R Rao* \\ Department of Laboratory Medicine and Pathology, University of Minnesota, USA
}

Submission: December 04, 2018; Published: January 29, 2019

*Corresponding author: Gundu H R Rao, Laboratory Medicine and Pathology, Director, Thrombosis Research, Lillehei Heart Institute University of Minnesota, Founder CEO, Global Alliance for Traditional Health Systems, Mind Body Spirit Society, Mysore, USA

\begin{abstract}
Global Burden of Disease (GBD) Group, has published a series of articles in the reputed journal of Lancet, in the last few years providing the readers, access to vast amount of statistical data, on the rapidly increasing Global Burden of Diseases across the Globe. In the area of cardiometabolic diseases, they report that all the known metabolic diseases, such as hypertension, excess weight, obesity, prediabetes, diabetes (type-2), and vascular diseases, have reached epidemic proportions. In spite of these observations and innumerable reports, hardly there is any major report, suggesting ways to stop, reduce, reverse, or prevent these chronic diseases. If these diseases are not checked in the near future, the economic disaster they cause, will be disastrous for the developing nations, which carry the major healthcare burden of these diseases. Framingham Heart Study group, which was initiated 70 years ago, by the prestigious National Institutes of Health (NIH) USA, developed a list of modifiable risks, that promote cardiovascular diseases. Several studies since then, have shown that managements of these modifiable risks, have contributed significantly, to the decline in deaths due to CVDs worldwide. However, there is no such clear-cut risk factors described, for other metabolic diseases. In view of this fact, no country has reduced, or reversed, the rate of increase of metabolic diseases in the last three decades. Since this article is about diabetes, we will focus on this particular disease and discuss our options to contain this disease. In majority of the countries, standard diabetes care seems to be, treatment of patients, with oral hypoglycemics, and then follow with insulin therapy, when oral medications fail or become inadequate. We will discuss in this overview, early risk factors for the development of metabolic diseases, starting from the time of conception, and available options for interventions. We also will discuss childhood obesity, oxidative stress, inflammation, endothelial dysfunction, and subclinical atherosclerosis, as possible causes of observed metabolic risks, and suggest complimentary therapies, for these observed risks. Finally, using India as a model for the developing countries, we will suggest a few options for implementing, populationbased prevention strategies.
\end{abstract}

Keywords: Global burden of disease; Cardiometabolic diseases; Metabolic diseases; Hypertension; Diabetes

\section{Introduction}

In the last three decades there is a rapid increase in cardiometabolic diseases such as hypertension, excess weight, obesity, type-2 diabetes and vascular diseases to epidemic proportions worldwide [1-13]. In the same period the incidence of type-2 diabetes has increased over 17-fold in China [14]. By and large, the reason for this global increase seems to be the rapid urbanization, ease in commuting, sedentary habits, unhealthy lifestyles and Western diet pattern. In view of this observation, there has been some attempts to initiate, global prevention strategies. With this goal in mind, United Nations General Assembly in October of 2018, during the Plenary Meetings of the Seventy-Third Session, adopted two resolutions, -General Assembly Reaffirms Political Declarations on Urgent Global Responses to Tuberculosis, Non-Communicable Diseases (NCDs). The assembly adapted the resolution, "political declaration of the third high-level meeting of the General Assembly on the prevention of non-communicable diseases." This declaration is nothing but the re-adaptation of the earlier declarations, which all member countries had signed. The representative of Italy, explaining her delegation's position said, that she had the honor to co-facilitate negotiation and that the declaration is concise, balanced, and builds on current opportunities. The resolution highlights the importance of healthy lifestyle choice for the prevention of non-communicable diseases. The highlevel committee "Approved Draft Declaration, World Leaders in General Assembly Pledged Increasing Multi Stakeholder, Multisectoral Response to Tackle Non-Communicable Diseases."

In a historic United Nations' (UNs) Summit on September 25, 2015, the world leaders adopted the 17 Sustainable Development Goals (SDGs) to be achieved by 2030. Three interconnected, core elements, namely economic growth, social inclusion, and environmental protection, are identified for sustainable and inclusive growth of all. In the past few decades, noncommunicable diseases (NCDs), have emerged as a major public health problem in the world, due to demographic, epidemiological, and socioeconomic transition. Recognizing the 
social, economic and public health impact of NCDs, the WHO Global NCD Action Plan 2013-2020 and nine voluntaries global NCD targets were adopted by the World Health Assembly in 2013. This document provides information about the voluntary global target, to halt the rise in diabetes by 2025 . Reaching this target is also an important step, toward realizing the commitments made at the UN General Assembly High-level Meetings on NCDs in 2011 and 2014, and the vision of the 2030 Agenda, for Sustainable Development and the Sustainable Development Goals (SDGs), including target 3.4 to reduce premature NCD mortality $[15,16]$ Considering the progress made in the last five years, since the adaptation of the SDG, the experts predict, that chances of stopping the increase in the incidence of diabetes worldwide by 2015 , is less than 1 percent. According to the experts, over 415 million people live with diabetes and estimated 193 million people have undiagnosed diabetes [17]. Early detection through screening programmes and the availability of safe and effective therapies, will reduce morbidity and mortality by preventing or delaying diabetes-related complications.

Traditional Medicine practitioners of India and China have used herbal medicine and various phytochemicals for centuries and reaped the benefits of their medicinal properties. However, the regulatory agencies of the US, the UK and EU will not allow the sales of these drugs as therapeutics. In the USA, Public Health Services established a National Center for Complementary and Alternative Medicine (NCCAM) in 1991. Over 50 US academic institutions, have complementary medicine programs funded by NCCAM. Dr Josephine Briggs the ex-director of this Institute says, "that the use of herbal medicine is not acceptable, as they do not meet the safety and efficacy criteria established by the US regulatory agencies (FDA). The name of this center has been changed to National Center for Complementary and Integrative Health (NCCIH). Now the question that rises in the minds of those, who want to promote integrated health programs is, how do we accomplish this goal without doing very expensive often confusing and contradicting large clinical trials? Since 1980, obesity has increased worldwide by two-fold and diabetes fourfold. Patients with diabetes, have a two to three-fold higher risk for developing cardiovascular disease and cerebrovascular diseases. How did we reach this sorry state, in spite of the tremendous improvements in health care worldwide? What other measures should we take, to reduce or prevent these chronic metabolic diseases? In this review, we will try to address some of the issues, and discuss some alternative, or complementary approaches. Since China and India, have highest number of subjects with diabetes, we will use these countries as model countries, for the development of an integrated approach, to the management of diabetes.

\section{Discussion}

Healthcare workers all over the world, are aware of the inadequacy of the western medicine, which is disease centric and the merits of integrated approach to health. In recognition of this awareness, National Insitutes of Health (NIH) USA dedicated a separate Institute, to address issues related to complementary and alternative medicine (CAM). However, the regulatory agencies in the USA, the UK, and the European Union, have banned the sales of herbal medicine in their respective countries, citing safety and efficacy issues. In recognition of the growing body of evidence about the importance of integrative approach to health, in 2009 Institute of Medicine (IOM), NIH, USA, organized a summit on Integrative Medicine and Health of the Public. More than 600 health care providers participated in this summit. The IOM summit summary states, "The disease-driven approach to care has resulted in spiralling costs as well as a fragmented health system, that is reactive and episodic, as well as inefficient and impersonal." In spite of these international efforts, no country has developed an integrated, holistic approach, to the management of cardiometabolic diseases, which have reached epidemic proportions worldwide. As we have mentioned before, western medicine is disease centric, as such has not been very effective, in the development of holistic or integrative approach, to the prevention of chronic diseases. On the other hand, traditional medicine, which claims holistic practice of medicine, is also competing for the same space, with allopathic clinicians and as such, are missing a great opportunity for developing robust preventive medicine approach for stopping, reducing, reversing, or preventing cardiometabolic diseases.

In China and India over the last century, traditional medicine has co-existed with allopathic medicine. There are 350,000 staff working at more than 2500 hospitals of traditional medicine in China. India has over 700,000 Ayurvedic practitioners, there is one primary health care center (PHC) for every 30,000 population, and 5 to 6 community health centers (CHCs) for each PHC. What we are trying to propose is a bold novel approach, to integrate some aspects of traditional medicine, with some allopathic methods and come up with a robust, holistic, integrated platform for the management of metabolic diseases. After all, the back bone of the Indian and Tibetan Traditional medicine is, "Dosha System of Classification of Altered Metabolism." Therefore, this approach provides a great opportunity, to put to test this ancient concept, and develop holistic approaches, to the management of chronic metabolic diseases $[18,19]$. When attempting to propose such an approach, it is important to take into consideration the existing infrastructure, human resources, and various state and national level initiatives. We also have to consider, as to what is the earliest metabolic alteration, that we need to address in this holistic integrated approach. Both in India as well as in China, there are some efforts to introduce health education in school curriculum, to promote physical activity and health eating habits. Complementary to this educational program for school going children, another program to empower women should also be initiated.

Published evidence shows, that 15 million of all deaths attributed to NCDs, occur between the ages of 38 and 69 years. Of these "premature" deaths, over $85 \%$ are estimated to occur, in low-and middle-income countries. Both China and India, share 
a common origin of NCDs. It has been very well established by decades of epidemiological studies, that more than $30 \%$ of the children born in Asian countries, are of low birth weight. These children are at risk, for developing cardiometabolic diseases, at later stages of adult life [20,21]. Harvard researchers have developed, multi nutrient supplements, to reduce this observed health burden [22]. Ayush trained clinicians, should be able to provide the needed consultations to "would be" parents, about the importance of micronutrients, and also recommend multinutrient supplements during this critical period in life, to prevent the fetal origin of adult diseases [23].

Recently AYUSH, the platform that is responsible for Traditional Medicine Education in India, announced "Ayush Therapy" at Government Centers, for borderline lifestyle ailments. All metabolic diseases, such as hypertension, excess weight, obesity, prediabetes, diabetes, and vascular diseases, are lifestyle diseases by definition. The type of program suggested by the AYUSH, makes a great sense, and if implemented properly, can revolutionize the way healthcare is provided in a country like India. The program announced by AYUSH envisages, establishment of this kind of therapy at the 201 NCD-centers across the state. We would like to see such program established, in all the 15, 2326 PHCs, in the 640 districts of the country. Furthermore, we also advocate the use of modern diagnostic tools and platforms, for the diagnosis of the various metabolic risks, so that the therapy can be initiated at the appropriate stages and the progression or regression of the risks managed properly. If we take this approach of early detection of the various metabolic risks, and management of observed risks, then we can hope to achieve the containment, or reduction of metabolic diseases in the near future.

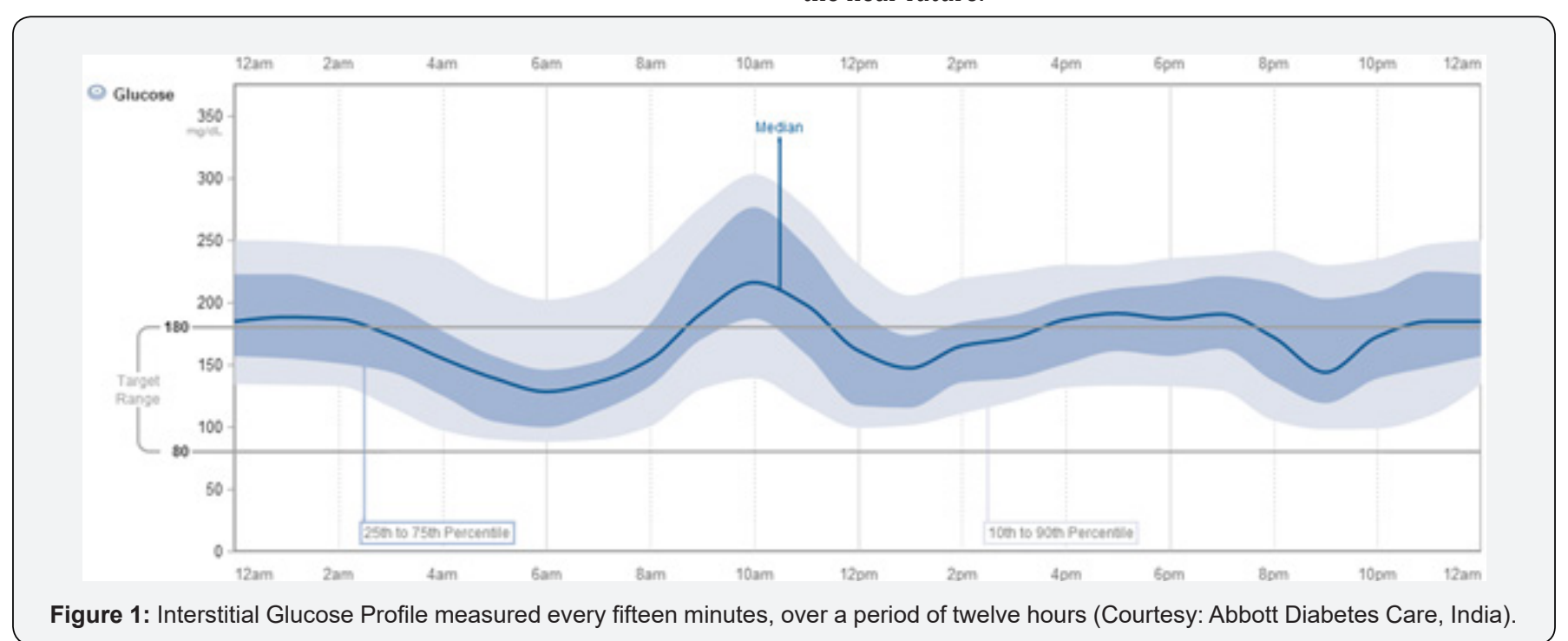

Next level of the preventive strategy should be, to look at the childhood obesity and prediabetes. The Asian phenotype has a different kind of obesity compared to the Caucasians. Therefore, looking at the body-mass index (BMI) may not be the ideal approach.Wehave demonstrated in our earlier studies, that Waist/ Hip ratio will be a better approach to monitor abdominal obesity than the BMI. At the same time, screening should be initiated to look at the fasting glucose, as well as postprandial glucose, to detect early onset of diabetes or pre-diabetic conditions. These types of tests will help in identifying individuals with borderline lifestyle disorders [24-26]. Detection at this stage in life, will help prevent, further progression of the diseases, or at least postpone the development of clinical complications. For the diagnosis of blood glucose levels, any standard over the counter glucose monitor may be sufficient. However, if the staff can be trained to use the continuous glucose monitors (CGMs), available in the market, such as Abbott FreeStyle Libre, or Dexcom G6, then one can develop simple methods, to measure interstitial glucose profiles, as well as to validate the efficacy or otherwise of a variety of anti-diabetic indigenous medical preparations, dietary components, and formulations. In a representative figure shown below, you can see the fluctuations, in the interstitial glucose levels over a period of 12 hours. The glucose levels are determined every fifteen minutes. In the Dexcom G6 device, the glucose is monitored every five minutes. Since one can access the data any time during study, one can follow the beneficial effect of it or lack of it, of any phytochemical (Eg: mulberry green tea or herbal products), or a variety of indigenous anti-diabetic preparations, dietary or nutritional therapies (Figure 1).

Leading herbal product developer Himalayan Drugs, of India, has an anti-diabetic drug called, Diabecon, which contains ingredients from: Gymnema sylvestre, Pterocarpus marsupium, Glycyrrhiza glabra, Casearia esculenta, Syzgmium cumini, Asparagus racemosus, Boerhavia diffusa, Sphaeranthus indicus, Tinsopora cordifolia, Swertia chirata, Tribulus terrestris, Phyllanthus amarus, Gmelina arborea, Gossypium herbaceum, Berberis aristat, Aloe vera, Triphala, Commiphora wightii, shilajeet, Momordica charantia, Piper nigrum, Ocimum sanctum, Abuilton indicum, Curcuma longa, Rumex maritimus. Whereas, another product Diasulin contains; Cassia auriculata, Coccinia indica, Curcuma longa, Embilica officianlis, Syzugium cumini, 
Trigonella foenum graecum, Azadrichta indica, Ficus racemosa, Aegle marmelos, Cinnamomum tamala. Some Ayurvedic products have Gurmar (Gymnema sylvestre) Karela (Momordica charantia) Pushkarmool (Inula recemosa), Jamun gutli (Syzygium cumini) Neem (Azardochta indica) Methika (Trigonella foenum gracecum) Guduchhi (Tinospora cordifolia). The advantage of using CGMs to monitor glucose profiles, is that one can follow the effect of any of the complementary or alternate therapies, for their supplementary effect, in addition to whatever therapy that they may be taking for the management of diabetes. Using such approach, we have done preliminary studies on mulberry green tea, which had antiglycemic properties.

In countries like China and India, the growing prevalence of maternal obesity, gestational obesity, childhood obesity, and early onset diabetes and prediabetes, lead to premature mortality and morbidity. Furthermore, in Asian phenotype, central abdominal obesity as measured by waist circumference is a strong predictor of diabetes. This visceral obesity is associated with abnormal levels of adipokines, low grade inflammation, and insulin resistance. Early- onset of diabetes, increases the risk of premature mortality and diabetes-related complications, due to long duration of the disease. In China, the Da Qing Diabetes
Prevention Program, demonstrated the benefits of lifestyle modification, in reducing the risk of progression from impaired glucose tolerance to diabetes. In 2010, the Ministry of Health launched a comprehensive NCD prevention and control program, including health education and promotion, early detection and treatment, and standardized disease management at the community level in 140 counties, in 30 provinces in China [27]. We would like to see a similar diabetes prevention program developed in India, at the community level (PHCs/CHCs), using trained healthcare workers, Ayush Vaidyas, and any other NGOs interested in such prevention programs. Any population-based prevention program, developed by India and China, with such large prediabetic population, can be used as model for other developing countries.

In this overview, we have discussed the benefits of using CGMs for monitoring glucose profiles, as well as to evaluate the effectiveness or otherwise of complementary therapies. We can also add other noninvasive, simple to use diagnostic devices, to this approach, for monitoring cardiometabolic diseases for risk stratification, risk management, and prevention strategies [28-31]. (LD-Products, Miami, Florida, USA: www.ldteck.com) (Figure 2 \& 3).

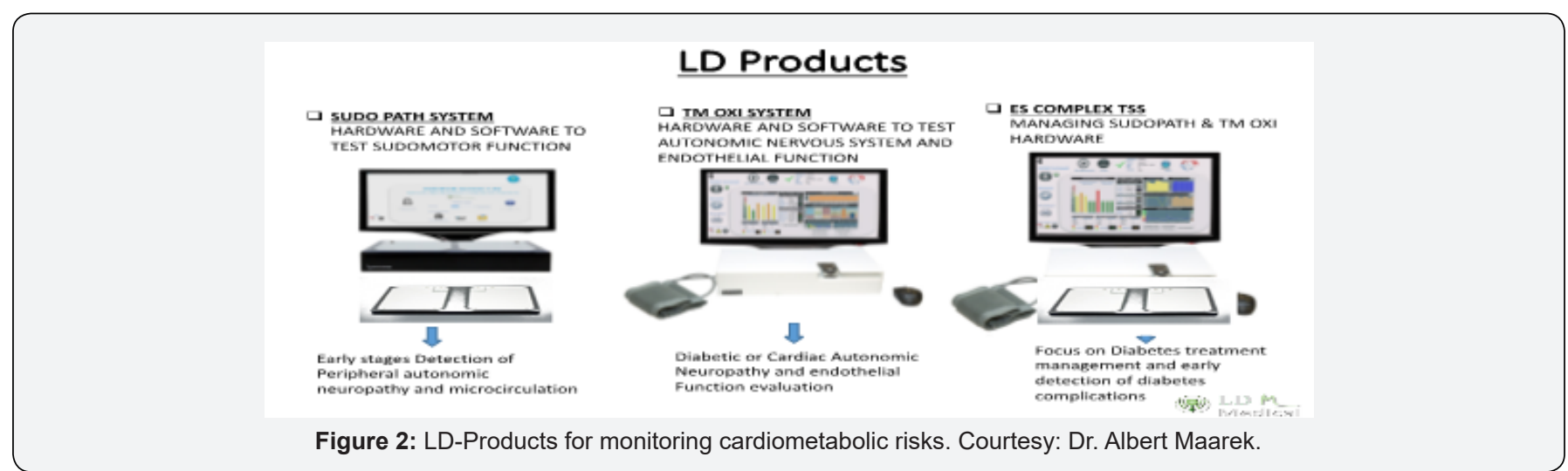

Figure 2: LD-Products for monitoring cardiometabolic risks. Courtesy: Dr. Albert Maarek.

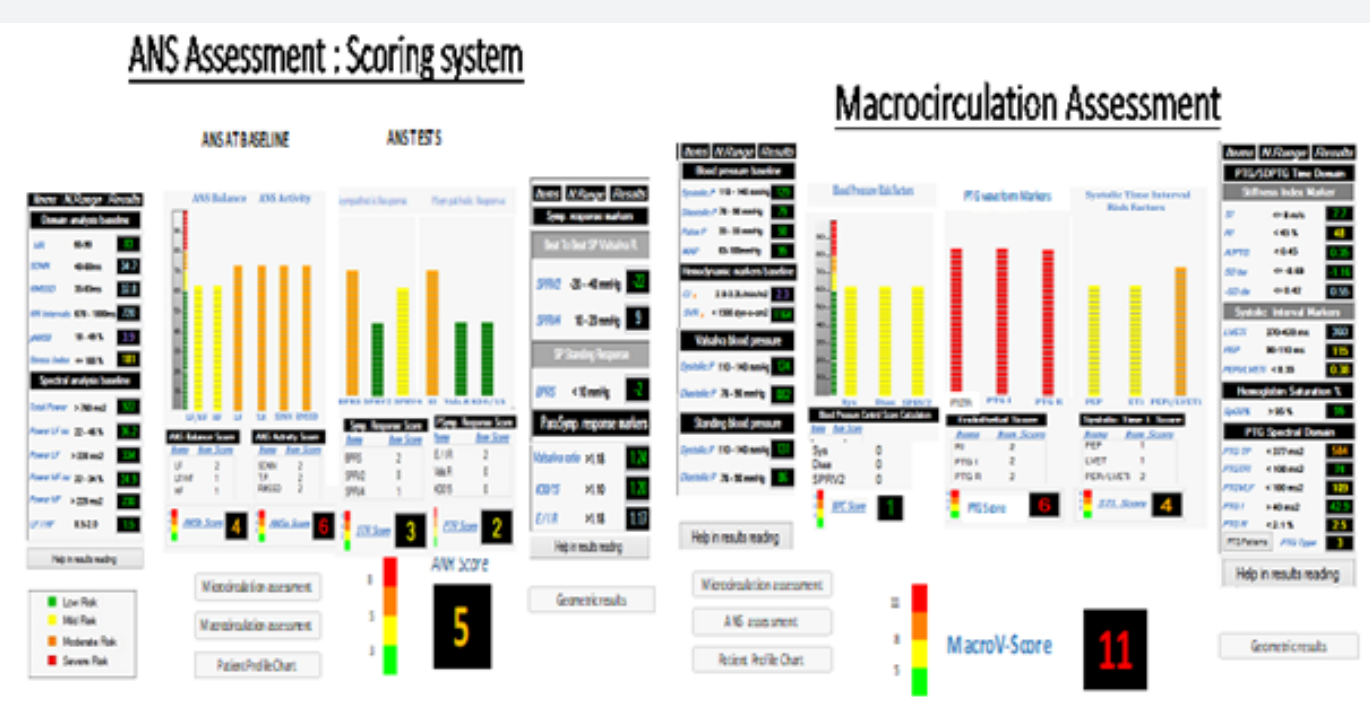

Figure 3: LD-Products for monitoring cardiometabolic risks. Courtesy: Dr. Albert Maarek. 
When planning a new novel and integrated approach to healthcare, we need to consider the disparity between personal choices, the differences that exist between the allopathic doctors, and the traditional medicine Viadyas. At the time of this writing, it is unthinkable that the West will accept traditional, complementary and alternative medicine (TCAM). In one form or the other, acupuncture, Ayurveda, Chinese medicine, are popularized in developing countries today, and seem to enjoy better attention, due to their popularity, integration, and holistic approach to healthy life. In this article, our emphasis is more on Integrative Medicine, than alternate or complementary medicine. Integrated medicine, - refers to certain evidencebased management, which uses best of both conventional and traditional medicine systems. It is quite evident, that Western medicine is disease centric, and has failed to prevent chronic metabolic diseases. Traditional medicine is patient centric, which claims to be holistic, yet, has failed to prevent the increase in the evidence of metabolic diseases. The reason being, in most of the developing countries, people do not go to seek medical help, unless they are sick. How do we integrate these systems, to achieve a balanced approach to health care, which aims at arresting, reducing, or reversing the increase in the incidence of metabolic diseases? It is important to ensure, that the knowledge and training of TCAM practitioners are adequate. It is also equally important, to develop a good understanding between the TCAM practitioners, and the conventional medicine practitioners, so that they understand the complementary nature of the Integrative Medicine. This brings us to the immediate need for change in the medical education system. In some countries TCAM courses are integrated into allopathic medical education. In others, the TCAM education also includes, teaching of some common topics, essential for understanding the essence of Integrated medicine.

According to experts in the area of TCAM, around $80 \%$ of the population continues to use the traditional medicine in Africa, Asia, and Latin America, and many governments in these regions, have been trying to incorporate traditional medicine practices, to help their primary health care needs. We have already mentioned some of the efforts, under consideration, in terms of diabetes education at various levels, development of awareness programs, and encouragement of personal level as well as population level prevention strategies. In view of the fact, that we are advocating the development of Integrated Medicine in developing countries, we also should emphasize the need for the development of guidelines and guidance statements, that would serve the needs of various healthcare providers. One size fit all, approach will not work. Furthermore, these guidelines, should be developed by the joint efforts of AYUSH experts and the Medical Council of India experts, taking into consideration that each group of practitioners, that is TCAM and allopathic, blend seamlessly in terms of providing Integrated care for these novel programs. These guidelines should consider, the need to empower, and encourage, the TCAM practitioners, the use of emerging tools and technologies, for diagnosis and management of risks, as well as for monitoring benefits or otherwise of the therapies. They should be made aware, that if the traditional medicine approach fails, the subjects should be advised to switch to conventional medicine therapies. Similarly, if subjects taking allopathic medicine, desire to try traditional therapies as complementary therapies, they should be allowed to do so under medical supervision.

It is easy to propose such novel, integrated approached to the healthcare. But it is not easy, to implement such a project without passion, dedication, and commitment of various stakeholders. Moreover, we need to build a different culture altogether, in which healthy people are counseled for lifestyle changes, and healthy living habits. In order to prevent the development of these chronic diseases, the screening for altered metabolism and counseling for the management of such alterations, should start early in life. Considering the large number of low birth babies born in these countries, newlyweds, should be counselled early on, about the need for supplemental micronutrients during pregnancy, to prevent fetal origin of adult diseases. Obesity to a large extent is the driving force, for increased incidence of diabetes. Therefore, all-out effort should be made, to treat obesity and obesity-related complications. Similarly, one can recognize symptoms of altered metabolism, leading to prediabetic state, and appropriate interventions should be developed to counsel these individuals, so that diabetes and its complications could be prevented or postponed.

In developing countries, where no one goes to a doctor unless one is sick, it is hard to get them, to any of these preventive programs. Just like there was a global effort, in the prevention of use of tobacco and tobacco products, a massive educational program should be developed to educate, create awareness, of the serious health issues related cardiometabolic diseases. Every member country of the United Nations has signed a declaration, to stop increase in the incidence of diabetes by 2030. These high-level committees also have made declaration to stop the increase in the increase of diabetes in 2025 to 2020 level. These are commendable goals [32-37]. Having said that, I want to mention, that the various expert committees working on such task forces, have reported that achieving such sustainable Million Development Goals (MDGs) are highly unlikely. Making declarations, developing guidelines, are celebrating World Diabetes Day, will not solve the problem of these epidemics. They can be solved only by commitment, and dedicated work by all the stakeholders. Furthermore, to be effective, such projects need trained human resources, healthcare infrastructure, and firm financial commitment. In a short overview like this, we will not be able to provide all the solutions and answers. We just have provided a view point, that can be considered by the policy makers and various governments. We would be glad to be a part of any such effort.

Recently, the Ministry of AYUSH decided to celebrate National Ayurveda Day on Dhanvantri Jayanti every year. According to a report on their website, the "Mission Madhumeha (Diabetes)" 
was launched on the occasion of first National Ayurveda Day, celebrated on 28th October 2016 [38]. In announcing this mission, following statement was issued by AYUSH: This protocol for "Prevention and Control of Diabetes through Ayurveda" has been prepared for implementing the "Mission Madhumeha". An attempt has been made to bring out the Ayurvedic philosophy in implementable format. The tables about do's and don'ts have been depicted with relevant figures for easy understanding of the community. The 'Madhumeha Assessment Tool' (MAT) based on Ayurvedic Philosophy has been developed for the first time for the self-assessment of people with regard to possibility of diabetes. The intention of developing the Madhumeha assessment tool is to sensitise people about diabetes, it's common symptoms, the Ayurvedic approach of Medodushti and its symptoms and to encourage them to visit an Ayurveda hospital, in case of having the score above the threshold. The specific diet and exercises explained in the protocol would be useful for the end user to promote self-health. The treatment/ medicines explained for implementation at PHCs/CHCs/ would provide, the base line for the insurance coverage purpose also. The charts have been developed in easy downloadable format, which could be used for developing, posters for display at places like Educational Institutes, Panchayat and such other relevant public places. Announcement of this protocol, and the plan to initiate NCD prevention through AYUSH efforts, are indication of the Government of India to strengthen primary care at PHCs/ CHCs. We are just expressing our view point on this topic of great public health importance and suggesting that they should not only develop Ayurveda approach to this problem, but also seriously consider the development, of "Integrative Medicine Approach", as suggested in this overview.

\section{Conclusion}

Cardiometabolic diseases have reached epidemic proportions worldwide. In the last three decades, obesity has increased by two-fold and diabetes by four-fold. In the same period in China, diabetes has increased by 17 -fold. It is hard to believe, that an advanced country like the USA, has the highest prevalence of diabetes. What is surprising is, the huge difference between the prevalence of diabetes in adults, versus prevalence of prediabetes. In China for instance, the estimate of the prevalence among adults is $11.6 \%$ and the prevalence of prediabetes is $50.1 \%$. Twin epidemics of obesity and diabetes is a rapidly growing public health menace, and if not checked soon, will result in economic disasters in several developing economies. In view of this fact, we are proposing an integrated approach to healthcare, which envisages the use of existing infrastructure, and trained human resources, and develop a seamless bridge between the traditional medicine and conventional medicine. India and China, which have such healthcare platforms, with some effort, could develop an integrated system of healthcare delivery, which will revolutionize the way healthcare is delivered worldwide.

As we mentioned in the article, there are some fractured attempts, to develop such healthcare in developing countries.
However, it needs more than declaration of efforts, or publishing protocols. It needs the will, dedication, passion, and commitment of all the stakeholders. We need to create a whole new culture, change attitudes of people, provide incentives, use multimedia platforms, digital infrastructures and smart Apps, to put together a seamless project, which provides opportunity for individuals to obtain the benefits of personal, customized health care. Such a system should integrate diagnostic tools, platforms and emerging technologies, for early detection of the risks, cluster of risks, and follow the effect of various therapeutic approaches. Without such integration, we will not be able to accomplish our goals of preventing reducing, reversing, or prevention of metabolic diseases. Guidelines, guidance statements, and protocols, should be developed by both AYUSH experts, as well as Medical Council of India, so that practitioners of both the systems of medicine, agree to use such a protocol. If we take this approach to health care, we can also develop collaboration with the Global Health Platforms, as well as the National Institutes of Health, USA. We have barely touched novel emerging approaches, to healthcare delivery. If developed properly, with evidence-based knowledge, these novel integrated approaches, could become the leading way of healthcare delivery worldwide.

\section{Acknowledgement}

Author expresses thanks to Dr. Poornima Murthy, Dr. Krishna Murthy, The Directors, Prajna Kuteera Ayurvedic Center, Mysore, India, and Dr. B. R. Pai, Founder Director, Vijay Foundation, Mysore, India, Co-Founders of the Global Alliance of Traditional Health Systems (GATHS), and the Mind Body Spirit Society of India, Mysore, for continued support and collaboration of my efforts, in developing Integrated Medicine Platforms in India.

\section{References}

1. Forouzanfar M, Liu P, Roth GA, Ng M, Biryukov S, et al. (2017) Global burden of hypertension and systolic blood pressure of at least 110 to 115 mmHg, 1990-2015. JAMA 317(2): 165-182.

2. Van Gaal LF, Mertens IL, De Block CE (2006) Mechanisms linking obesity with cardiovascular disease. Nature 444(7121): 875-880.

3. GBD 2015 Risk Factors Collaborators (2016) Global, regional, and national comparative risk assessment of 79 behavioral, environmental and occupational, and metabolic risk or clusters of risks, 1990-2015: a systematic analysis for the Global Burden of Diseases Study 2015 Lancet 388(10053): 1659-1724.

4. Zheng X, Jin C, Liu Y, Zhang J, Zhu Y, et al. (2015) Arterial stiffness as a predictor of clinical hypertension. J Clin Hyperten (Greenwich) 17(8): 582-291.

5. Ng M, Fleming T, Robinson M, Thomson B, Graetz N, et al. (2014) Global, regional, and national prevalence of overweight and obesity in children and adults during 1980-2013: a systematic analysis for the Global Disease Study 2013 Lancet 384(9945): 766-781.

6. Lu Y, Hajifathalian K, Ezzati M, et al. (2014) Metabolic mediators of the Risk Factors for Chronic Diseases Collaborations (BMI Mediated Effects). Metabolic mediators of the effect of body-mass index, overweight and obesity on coronary heart disease and stroke: a systematic analysis of 97 prospective cohorts with 1.8 million participants. Lancet 383(9921): 970-983.

7. Zommet P, Magliano D, Matsuzawa Y, Alberti G, Shaw J (2005) The metabolic syndrome: a global public health problem and a new definition. J Atheroscler Thromb 12(6): 295-300. 
8. (2016) Global Report on Diabetes: World Health Organization 2016. (1 Diabetes Mellitus (DM)-Epidemiology. 2. DM-prevention and control. 3 Diabetes, Gestational. 4. Chronic Diseases. 5. Public Health.).

9. (2016) Worldwide trends in diabetes since 1980: a pooled analysis of 751 population-based studies with 4.4 million participants. Lancet 387(10027): 1513-1530.

10. Mohan V, Rao GHR (2007) Type-2 diabetes in South Asians: Epidemiology, Risk factors, and Prevention. JP Medical Publishers, New Delhi, India.

11. Rao GHR, VJ Kakkar (1999) Coronary Artery Disease in South Asians. JP Medical Publishers, New Delhi, India.

12. Rao GHR, Thanikachalam S (2005) Coronary Artery Disease. JP Medical Publishers, New Delhi, India. 2005.

13. Rao GHR (2016) Coronary Artery Disease. MacMillan Medical Communications/ Springer/Nature. India.

14. Shen X, Vaidya A, Wu S, Gao X (2016) The diabetes epidemic in China: An integrated review of National Surveys. Endocrinol Pract 22(9): $1119-1120$

15. NCD Risk Factor Collaboration (NCD-RisC) (2017) Worldwide trends in body mass index, underweight, overweight, and obesity from 1975 to 2016: a pooled analysis of 2416 population-based measurements studies in 128-9 million children, adolescents, and adults. Lancet 390(10113): 2627-2642.

16. NCD Risk Factor Collaboration (NCD-RisC) (2016) Worldwide trends in diabetes since 1980: a pooled analysis of 751 population-based studies with 4.4 million participants. Lancet 387: 1513-1530.

17. Chaterjee S, Khunti K, Davies M (2017) Type-2 diabetes. Lancet 398 2239-2251.

18. Rao GHR (2015) Integrative approach to health: Challenges and Opportunities. J Ayur Integr Med 6(3): 215-219.

19. Rao GHR (2017) Opportunities and Challenges in Ayurveda: Global Perspective. Altern \& Integr Med 6: 239.

20. Rao GHR, Gandhi PG, Sharma V (2014) Clinical complications of Type2 diabetes mellitus in South Asians and Chinese populations: An Overview. J Diab Metab 5:240.

21. Xiao X, Zhang ZX, Li WH, et al (2010) Low birth weight is associated with components of the metabolic syndrome. Metabolism 59(9): 12821286.

22. Kawai K, Spiegelman D, Shankar AH, et al (2011) Maternal multiple micronutrient supplementation and pregnancy outcomes in developing countries: meta-analysis and meta-regression. Bull. World Health Organ 89(6): 402B-411B.

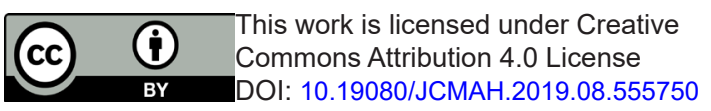

23. Calkins K, Devaskar SU (2011) Fetal Origin of adult diseases. Curr Probl Pediatr Adolesc Health Care 41(6): 158-176.

24. Rao GHR (2018) Integration of novel emerging technologies for the management of type-2 diabetes. Arch Diab \& Obesity 1(1).

25. Rao GHR (2018) Diagnosis of early risks, management of risks, and reduction of vascular diseases. J Clin Card \& Diagnostics 1(1): 1-11.

26. Rao GHR (2018) Nontraditional Approaches to the Management of Diabetes-related Complications. EC Endocrinol. Metab Res 3(5): 177-185.

27. Yin J, Kong APS, Chan JCN (2016) Prevention and care programs addressing the growing prevalence of diabetes in China. Curr Diab Reports 16(12): 130 .

28. Gandhi PG, Rao GHR (2014) The Spectral Analysis of Photoplethysmography to Evaluate an Independent Cardiovascular Risk Factor. Int J Gen Med 7: 539-547.

29. Gandhi PG, Rao GHR (2015) Detection of Neuropathy Using Sudomotor Tests in Type-2 Diabetes. Degenerative Neurological and Neuromuscular Diseases 5: 1-7.

30. Maarek A, Gandhi PG, Rao GHR (2015) Identifying autonomic neuropathy and endothelial dysfunction in Type 11 diabetic patients. EC Neurology 22: 63-78.

31. Rao GHR, Gandhi PG (2014) Need for a non-invasive diagnostic platform for early detection and management of cardiometabolic disorders. J Clin Prevent Cardiol 3(3): 93-98.

32. Nugent R, Bertram MY, Jan S (2018) Investing in non-communicable disease prevention and management to advance the sustainable development goals. Lancet 391: 2029-2035.

33. Stenberg K, Nanssen 0, Edejer TT. (2017) Financing transformative health systems towards achievement of the health sustainable development goals: a model for projected resource needs in 67 low-income and middle-income countries. Lancet Global Health 5: e875-887.

34. WHO (2016) Framework on integrated people-centered health services.

35. Taggart J, Williams A, Dennis S (2012) A systematic review of interventions in primary care to improve health literacy for chronic disease behavioral risk factors. BMC Fam Pract 13: 49.

36. UN General Assembly: Political declaration of the high-level meeting of the General Assembly on the prevention and control of non-communicable diseases. United Nations, New York 2011:13.

37. Horton R (2017) Offline: NCDs-why are we failing? Lancet 390: 346. 38. Protocol for prevention and control of Diabetes through Ayurveda.

\section{Your next submission with Juniper Publishers will reach you the below assets}

- Quality Editorial service

- Swift Peer Review

- Reprints availability

- E-prints Service

- Manuscript Podcast for convenient understanding

- Global attainment for your research

- Manuscript accessibility in different formats

( Pdf, E-pub, Full Text, Audio)

- Unceasing customer service

Track the below URL for one-step submission

https://juniperpublishers.com/online-submission.php 